\title{
Des protéines à motifs riches en leucines définissent une cinquième famille de molécules d'adhérence
}

De nombreuses protéines ont un domaine constitué d'un nombre variable de répétitions d'une série d'acides aminés comportant plusieurs résidus leucine espacés régulièrement. Ces molécules exercent leur activité à différents niveaux dans la cellule. Certaines d'entre elles, comme GPIb chez l'homme, la chaoptine, Toll ou la connectine chez la drosophile, semblent jouer un rôle de molécules d'adhérence dans des processus nécessitant des contacts intercellulaires ou avec la matrice extracellulaire. GPIb fait partie d'un complexe moléculaire essentiel pour l'adhérence des plaquettes au sous-endothélium. La chaoptine, Toll et la connectine sont diversement impliquées au cours du développement de la mouche.

\section{Vincent Ollendorff Tetsuro Noguchi Daniel Birnbaum}

\section{ADRESSES}

V. Ollendorff: docteur de l'université de Paris VII. T. Noguchi : docteur de l'université d'Aix-Marseille II. D. Birnbaum : directeur de recherche à l'Inserm. Laboratoire d'oncologie

moléculaire, U. 119 Inserm, 27, boulevard Leï-Roure, 13009 Marseille, France. 'adhérence de cellules entre elles, ou avec la matrice extracellulaire par l'intermédiaire de molécules spécifiques est une composante fondamentale de la communication cellulaire. C'est un processus critique au cours du développement et de la différenciation de divers tissus. Les molécules d'adhérence interviennent dans les interactions cellulaires, le contrôle des migrations cellulaires ou axonales et participent à la cohésion interne des organes. La possibilité pour une cellule d'exprimer de telles protéines conditionne en partie sa destinée en modulant sa capacité migratoire et ses interactions avec les cellules environnantes, interactions dont peuvent découler l'engagement dans une voie de différenciation ou des réponses physiologiques variées (réponse lymphocytaire, hémostase, cicatrisation...). Les contacts cellulecellule et cellule-matrice extracellulaire sont exercés par différents types de molécules d'adhérence qui montrent des spécificités cellulaires plus ou moins grandes. A ce jour, quatre grandes familles de molécules d'adhérence ont ainsi été définies sur des critères de similarités structurales et fonctionnelles : ce sont les intégrines, les molécules de type CAM appartenant à la superfamille des immunoglobulines, les sélectines, et les cadhérines ([1-4], pour revues).

\section{Adhérence cellulaire : la cinquième famille}

Une cinquième famille de molécules 
Tableau I

LA FAMILLE DES PROTÉINES A MOTIFS RICHES EN LEUCINES

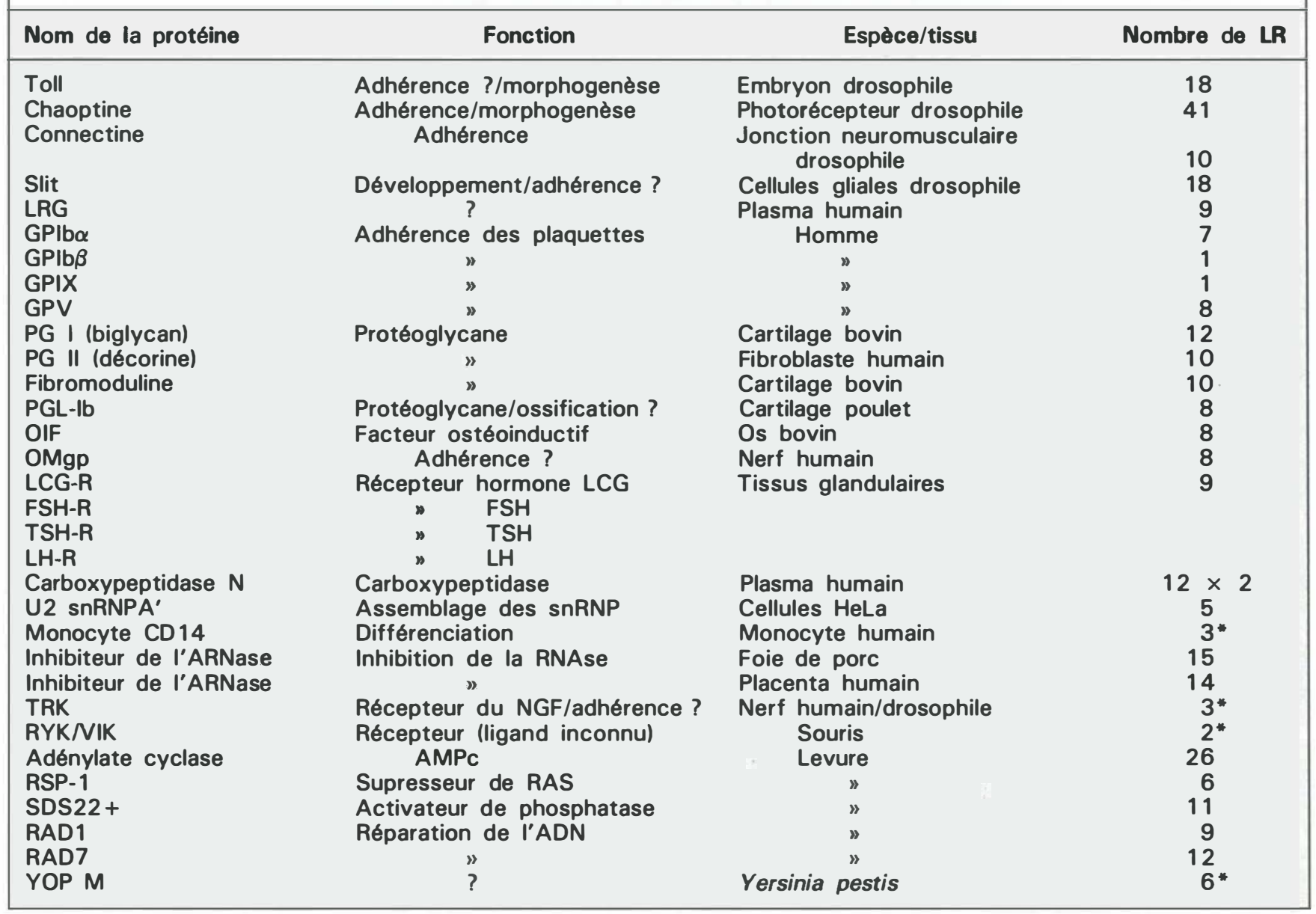

* Motifs à LR non canoniques.

d'adhérence émerge aujourd'hui d'un large groupe de protéines caractérisées par la présence d'un nombre variable de motifs riches en leucines (appelé motif LR) (Tableau I). Depuis quelques années, le nombre de ces protéines à motifs LR n'a cessé régulièrement de s'accroître. Ces polypeptides sont impliqués dans des processus biologiques très divers. Ils peuvent en effet occuper différents maillons d'une chaîne de transduction du signal, ou contribuer à des phénomènes d'adhérence cellulaire. Certains ont un profil d'expression restreint à un type cellulaire particulier tandis que d'autres sont exprimés dans un grand nombre de tissus. Avant de décrire plus spécifiquement les molécules d'adhérence qui en font partie, il faut présenter la super-famille de $\mathrm{m} / \mathrm{s} n^{\circ} 10$ vol. 9 , octobre 93 protéines à motifs LR. Une synthèse propre pourrait d'ailleurs être consacrée à cette super-famille si la tâche n'était rendue difficile par la diversité des molécules qui la composent.

\section{Le motif $L R$ : un rôle clé dans les interactions protéiques et un succès de l'évolution}

Le nombre de modules LR varie selon les protéines de un, pour GPIX chez l'homme, à 41 pour la chaoptine chez la drosophile. La cassette LR canonique se compose d'un espacement périodique de résidus leucines séparés par des acides aminés hydrophiles et hydrophobes occupant chacun des positions bien définies (figure 1). En particulier, une aspara- gine est pratiquement toujours présente à la même place au sein du motif. Certaines positions peuvent, en revanche, être occupées par un résidu quelconque. Des études portant sur la configuration d'un peptide LR consensus ont montré qu'il pouvait adopter une structure générale de type feuillet $\beta$ amphipathique [5].

Une trentaine de protéines distinctes comportant des motifs LR ont à ce jour été caractérisées. Elles proviennent aussi bien d'organismes unicellulaires (Yersinia pestis, Saccharomyces cerevisiae) que pluricellulaires (insecte, mammifères) (Tableau I). Retrouver une telle conservation dans des organismes si différents suggère qu'il existe des contraintes structurales importantes liées à la fonction de ces motifs LR. En outre, l'étendue des 


\section{RÉFÉRENCES}

1. Hynes R, Lander A. Contact and adhesive specificities in the associations, migrations, and targeting of cells and axons. Cell $1992 ; 68: 303-22$

2. Fischer A. Quatre familles de molécules responsables de l'adhérence intercellulaire. médecine/sciences $1991 ; 7$ : 540-2.

3. Mège RM. Les molécules d'adhérence cellulaire : molécules morphogénétiques. médecine/sciences $1991 ; 7: 544-52$.

4. Peyriéras N. Les mécanismes moléculaires de l'adhérence cellulaire. médecine/sciences $1992 ; 8: 591-6$.

5. Krantz DD, Zidovetski R, Kagan B, Zipursky SL. Amphipathic $\beta$ structure of a leucine-rich repeat peptide. J Biol Chem 1991 ; $256: 16801-7$.

6. Braun $T$, Schofield PR, Sprengel R. Amino-terminal leucine-rich repeats in gonadotropin receptors determine hormone selectivity. EMBO J 1991; 7 : 1885-90.

7. Kjellén L, Lindahl U. Proteoglycans : structures and interactions. Annu Rev Biochem $1991 ; 60: 443-75$

8. Andrews R, Fox J. Platelet receptors in hemostasis. Curr OP Cell Biol 1990; 2 : 894-901.

9. Roth G. Platelets and blood vessels : the adhesion event. Immunol Today $1992 ; 13$ : 100-5

10. Fox J, Berndt M. Cyclic AMP-dependent phosphorylation of glycoprotein Ib inhibits collagen-induced polymerization of actin in platelets. J Biol Chem 1989 ; 264 : 9520-6.

11. Mikol D, Gulcher J, Stefansson K. The oligodendrocytc-myelin glycoprotein belongs to a distinct family of proteins and contains the HNK-1 carbohydrate. J Cell Biol 1990 ; 110: 471-9.

12. Künemund $V$, Jungalwala $F$, Fisher $G$, Chou D, Keilhauer G, Schachner M. The L2/HNK-1 carbohydrate of neural cell adhesion molecules is involved in cell interactions. $J$ Cell Biol 1988 ; $106: 213-23$.

13. Schneider R, Schweiger M. A novel modular mosaic of cell adhesion motifs in the extracellular domains of the neurogenic trk and trkB tyrosine kinase receptors. Oncogene $1991 ; 6: 1807-11$.

14. Pulido D, Campuzano S, Koda T, Modolell J, Barbacid M. Dtrk, a Drosophila gene related to the trk family of ncurotrophin receptors, encodes a novel class of neural ccll adhesion molecule. EMBO J 1992; 11: 391-404.

15. Hovens C, Stacker S, Andres AC, Harpur A, Ziemiecki A, Wilks A. RYK, a receptor tyrosine kinase-related molecule with unusual kinase domain motifs. Proc Natl Acad Sci USA $1992 ; 89: 11818-22$.

16. Hortsch M, Goodman C. Cell and substrate adhesion molecules in Drosophila. Annu Rev Cell Biol 1991; 7 : 505-57.

17. Reinke R, Krantz D, Yen D, Zipursky L. Chaoptin, a cell surface glycoprotein required for Drosophila photoreceptor cell morphogenesis, contains a repeat motif found in yeast and human. Cell 1988; $52: 291-301$.

18. Van Vactor D, Krantz DD, Reinke R, Zipursky SL. Analysis of mutants in chaoptin, a photoreceptor cell-specific glycoprotein in Drosophila, reveals its role in cellular morphogenesis. Cell $1988 ; 52: 281-90$.

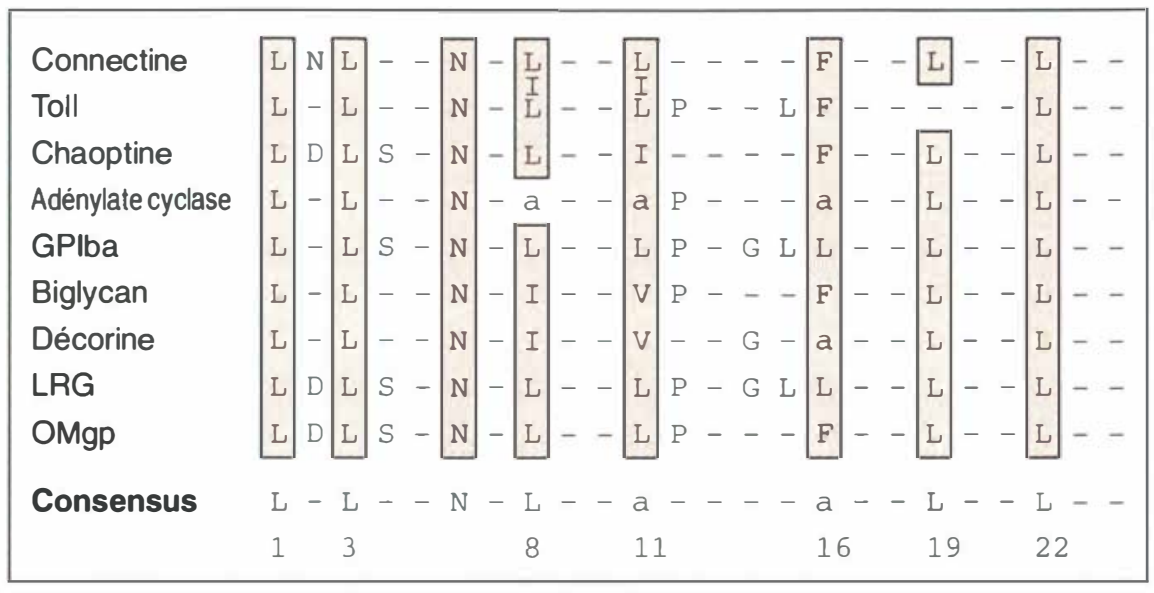

Figure 1. Séquences d'acides aminés des motifs riches en leucines. Les séquences consensus de différentes protéines sont alignées. Les positions conservées dans la majorité des molécules sont entourées et indiquées en bas. Les positions où l'on retrouve fréquemment des résidus leucines sont numérotées. $a=L, I, V$ ou $F$. Les positions 8 et 11 des motifs LR de la connectine sont de façon égale $L$ ou $\mathrm{l}$. Code à une lettre des acides aminés: $A$ : Ala ; C : Cys ; $D$ : Asp ; $E$ : Glu ; F : Phe ; G : Gly ; H : His ; I:Ile ; K : Lys ;

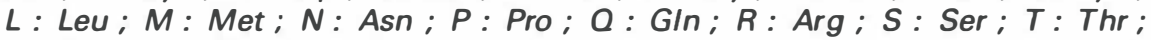
$V: V a l ; W: T r p ; Y: T y r ; X: n^{\prime i m p o r t e ~ q u e l ~ a c i d e ~ a m i n e ́ . ~}$

régions conservées dépasse souvent les seules répétitions LR et intéresse également les portions adjacentes à ces motifs. Ces segments voisins ont d'ailleurs pu jouer un rôle dans la duplication en tandem des motifs LR. Ils semblent, de plus, remplir une fonction capitale pour la structure tridimensionnelle de la protéine puisqu'ils comprennent des résidus cystéines impliqués dans des ponts disulfures intramoléculaires. La séquence d'un motif LR s'étend en moyenne sur 24 résidus, mais cette taille est sujette à variations selon les protéines (de 22 à 28 résidus). Cela tend à refléter une certaine plasticité du motif, suivant la fonction spécifique de la protéine. Le module LR est souvent associé à des motifs d'un autre type au sein de la même molécule. L'utilisation de combinaisons variées de domaines confère certainement une spécificité d'action à ces protéines à motifs LR. L'adénylate cyclase de levure est à ce titre la parfaite illustration de ce type de protéine mosaique associant, dans ce cas particulier, des motifs LR à un domaine catalytique.

\section{Une grande variété de fonctions pour les protéines à motifs $L R$}

Les fonctions des molécules de cette famille varient suivant leur localisation subcellulaire. Les protéines à motifs LR peuvent être membranaires, occuper une position intracellulaire où elles joueront un rôle de protéine régulatrice sous forme d'enzyme (comme les inhibiteurs de ribonucléase), ou de chaînon le long d'une voie de transduction (comme RSP-1, un suppresseur de RAS), ou bien encore être sécrétées, telles la carboxypeptidase $\mathrm{N}$ ou la protéine de drosophile Slit. Ces potentialités variées reposent néanmoins toujours sur la nécessité pour ces polypeptides d'exercer leur action par une interaction spécifique avec une autre protéine. Sa structure particulière et sa présence dans des protéines très distinctes, d'une part, sa conservation au cours de l'évolution, d'autre part, suggèrent un rôle neutre de connecteur pour le motif LR. Les molécu- 
les à motifs LR localisées à la surface des cellules sont de plusieurs types. Ce sont des récepteurs, des protéoglycanes, ou des molécules impliquées dans des phénomènes d'adhérence cellulaire. C'est par le truchement de leur domaine extracellulaire comportant les motifs LR que les récepteurs des hormones LH/CG, TSH et FSH fixent spécifiquement l'hormone adéquate [6]. Les protéoglycanes à motifs LR sont de petits glycosaminoglycanes localisés dans la matrice extracellulaire. Ce sont le biglycan, la décorine et la fibromoduline. Ils jouent des rôles importants dans diverses interactions, en particulier avec des facteurs de croissance [7].

Nous nous bornerons désormais à décrire les molécules de la famille à motifs LR qui ont une fonction adhésive démontrée ou supposée. Il s'agit de GPIb $\alpha$ et OMgp chez l'homme, et de la chaoptine, la connectine et la protéine Toll chez la drosophile. Les récepteurs à tyrosine kinase codés par les gènes $T R K$, à la fois chez l'homme et chez la drosophile, seront également évoqués.

\section{Les molécules à motifs LR dont le rôle dans I'adhérence est mal compris ou seulement supposé}

\section{Le complexe GPIb dans l'adhérence plaquettaire}

La lésion d'un vaisseau sanguin déclenche deux processus complémentaires : l'agrégation plaquettaire et la coagulation du plasma. Ces deux systèmes de régulation de l'hémostase sont néanmoins employés différemment selon le type de vaisseau affecté : les plaquettes interviennent surtout dans les lésions artérielles où le flux sanguin est élevé, alors que la coagulation du plasma est une réponse prépondérante en cas de blessure du réseau veineux. Si le processus de coagulation plasmatique résulte d'une série d'étapes enzymatiques conduisant à la production de thrombine et de fibrine, la réponse plaquettaire se décompose, elle, en trois événements séquentiels : l'adhérence, l'activation puis l'agrégation plaquettaire.

Dans la circulation artérielle, les plaquettes doivent tout d'abord s'arrêter puis se fixer à l'endroit endommagé du vaisseau. Cette adhérence cellulaire primaire crée un point d'ancrage favorable à l'addition d'autres plaquettes [8, 9]. Le contact entre les plaquettes et le subendothélium de l'artère lésée s'effectue par une interaction spécifique entre le facteur de von Willebrand ( $v W F$ ) et son récepteur plaquettaire GPIb. Ce dernier est un hétérodimère constitué de deux glycoprotéines : GPIb $\alpha$ et GPIb $\beta$. Ces deux polypeptides membranaires sont liés du côté extracellulaire par un pont disulfure. L'ensemble s'associe à la molécule GPIX de façon non covalente. La majeure partie de ces trois glycoprotéines (GPIb $\alpha$, GPIb $\beta$ et GPIX) est

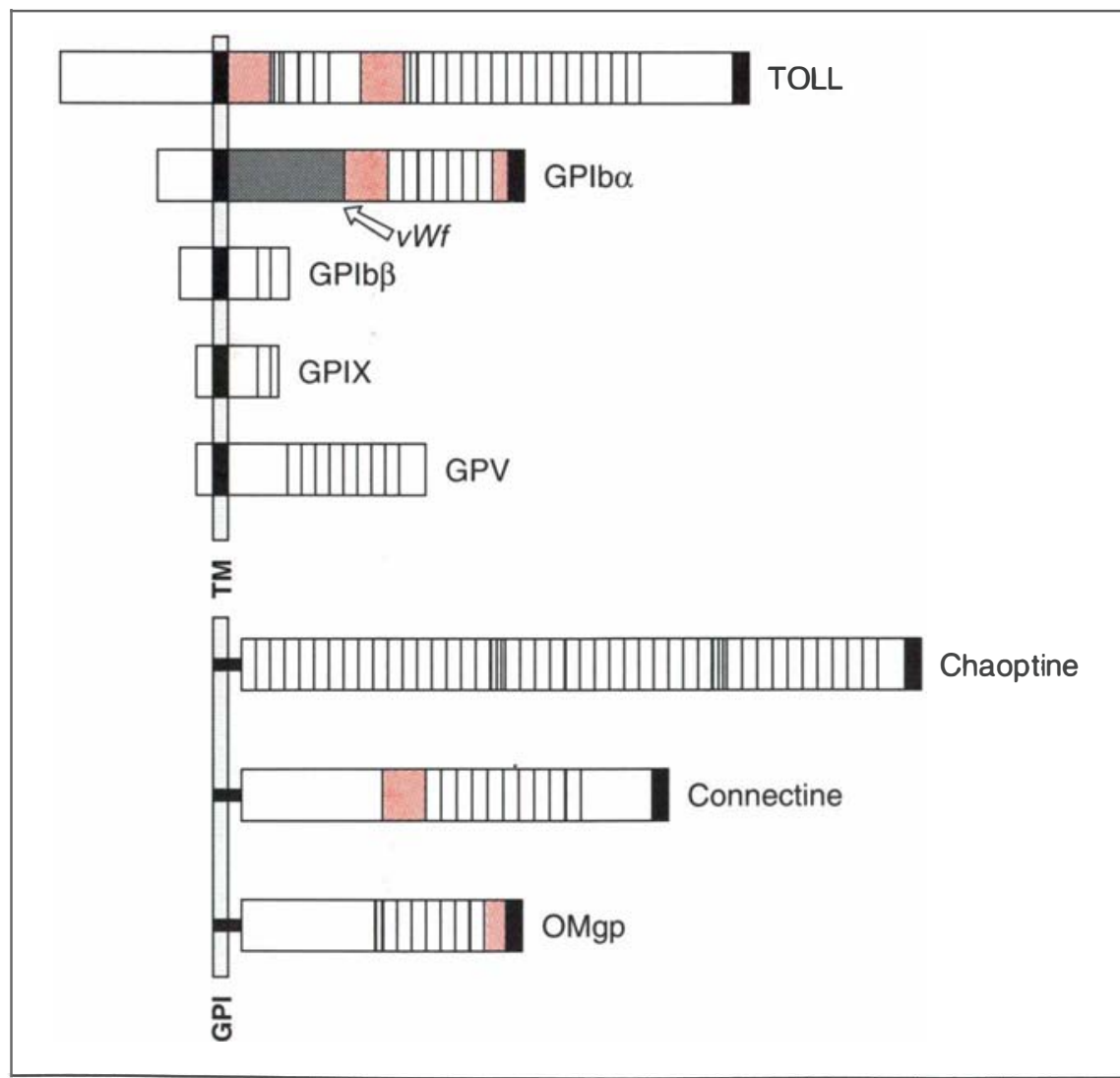

$\mathrm{m} / \mathrm{s} \mathrm{n}^{\circ} 10 \mathrm{vol} .9$, octobre 93
Figure 2. Représentation schématique de protéines membranaires à motifs riches en leucines. Deux types de protéines sont représentés: les protéines transmembranaires (TM) (Toll, GPIb $\alpha$, GPIb $\beta$, GPIX et GPV) et les protéines ancrées à la membrane par une structure de type glycosylphosphatidylinositol (GPI) (chaoptine, connectine et OMgp). Le peptide signal et le domaine transmembranaire sont représentés en noir ; les motifs riches en leucines sont représentés par des rectangles vides; ils peuvent être séparés par des portions moins homologues (rayures); les portions rosées indiquent les régions flanquantes qui présentent des similitudes de structure. Le domaine de GPIb $\alpha$ riche en résidus glycosylés est également représenté (grisé) ; le facteur de Von Willebrand (vWf) se fixe entre ce domaine et le domaine riche en leucines (flèche). 


\section{RÉFÉRENCES}

19. Krantz DD, Zipursky SL. Drosophila chaoptin, a member of the leucine-rich repeat family, is a photoreceptor cell-specific adhesion-molecule. $E M B O J 1990 ; 9$ : 1969-77.

20. Panthier JJ. Les récepteurs à tyrosine kinase et le développement embryonnaire. médecine/sciences $1992 ; 8: 1106-9$.

21. Tomlinson A. Cellular interactions in the developing Drosophila eye. Development 1988 ; 104: 183-93.

22. Zipursky SL, Venkatesh T, Teplow D, Benzer S. Neuronal development in the Drosophila retina : monoclonal antibodies as molecular probes. Cell 1984; 36 : 15-26.

23. Heguy A, Baldari C, Macchia G, Telford J, Melli M. Amino acids conserved in interleukin-1 receptors and the Drosophila Toll protein are essential for IL1-R signal transduction.J Biol Chem 1992 ; 267 : 2605-9. 24. Hashimoto C, Hudson K, Anderson K. The toll gene of Drosophila, required for dorsal-ventral embryonic polarity, appears to encode a transmembrane protein. Cell 1988 ; 52 : 269-79

25. Gerttula S, Jin Y, Anderson K. Zygotic expression and activity of the Drosophila Toll gene, a gene required maternally for embryonic dorsal-ventral pattern formation. Genetics $1988 ; 119: 123-33$.

26. Norris J, Manley J. Selective nuclear transport of the Drosophila morphogen dorsal can be established by a signaling pathway involving the transmembrane protein Toll and protein kinase A. Genes Dev 1992; 6 : 1654-67.

27. Keith F, Gay N. The Drosophila membrane receptor Toll can function to promote cellular adhesion. EMBO J $1990 ; 9$ : 4299-306.

28. Schneider D, Hudson K, Lin TY, Anderson K. Dominant and recessive mutations define functional domains of Toll, a transmembrane protein required for dorsalventral polarity in the Drosophila embryo. Genes Dev 1991 ; 5 : 797-807.

29. De Lotto R, Spierer P. A gene required for the specification of dorsal-ventral pattern of Drosophila appears to encode a serine protease. Nature $1986 ; 323$ : 688-92.

30. Chasan R, Anderson K. The role of easter gene, an apparent serine protease, in organizing the dorsal-ventral pattern of Drosophila embryo. Cell 1989 ; 56 : 391-400.

31. Nose A, Mahajan V, Goodman C. Connectin : a homophilic cell adhesion molecule expressed on a subset of muscles and the motoneurons that innervate them in Drosophila. Cell 1992 ; 70 : 553-67.

32. Gould A, White R. Connectin, a target of homeotic gene control in Drosophila. Development $1992 ; 116: 1163-74$.

33. Ollendorff V, Szepetowski P, Mattéi MG, Gaudray $\mathrm{P}$, Birnbaum D. New gene in the homologous human 11q13-q14 and mouse $7 \mathrm{~F}$ chromosomal regions. Mammalian Genome 1992 ; 2 : 195-200.

34. Low M. Glycosyl-phosphatidylinositol : a versatile anchor for cell surface proteins. FASEB J 1989 ; 3 : 1600-8

35. Ferguson $\mathbf{M}$, Williams A. Cell-surface anchoring of proteins via glycosylphosphatidylinositol structures. Annu Rev Biochem $1988 ; 57: 285-320$.

36. Stockdale F. Myogenic cell lineages. Dev Biol 1992 ; 154 : 284-98.

37. Burden S. Synapse-specific gene expres- sous forme complexée. Chacune d'elles possède un nombre variable de motifs LR : sept pour GPIb $\alpha$, un seul pour GIPb $\beta$ et GPIX. La déficience génétique en vWF (syndrome de von Willebrand) ou en GPIb (syndrome de Bernard Soulier) conduit à une absence d'adhérence plaquettaire. Dans le syndrome de Bernard Soulier, quatre protéines sont en fait absentes de la surface des plaquettes : GPIb $\alpha, \operatorname{GPIb} \beta$, GPIX et GPV. Cette dernière molécule comporte une dizaine de répétitions du motif LR. GPV s'associerait au complexe GPIb/GPIX et serait un substrat de la thrombine. On ne connaît pas les causes moléculaires expliquant l'absence combinée de ces quatre glycoprotéines à motifs LR, mais on peut envisager un défaut dans la coordination de leur régulation.

Au moment du contact avec le vaisseau endommagé, le vWF sert de lien entre le subendothélium et la surface des plaquettes. Ce facteur se fixe d'une part au collagène de type IV du compartiment subendothélial, et d'autre part au niveau d'un site spécifique de GPIb $\alpha$ compris entre le domaine à motifs LR et une région fortement glycosylée. Ce site de fixation de vWF se situe dans une portion de GPIb $\alpha$ particulièrement conservée dans la famille des molécules transmembranaires à LR (figure 2). Cette région comprend notamment quatre cystéines qui formeraient deux ponts disulfures intrachaînes, créant une boucle susceptible d'interagir avec le vWF. Le processus d'adhérence est déclenché par la force de cisaillement engendrée par un flux sanguin élevé [8, 9]. Cette force mécanique, en affectant les molécules de surface des plaquettes, rendrait accessible le site de fixation du vWF, ce qui permettrait l'adhérence des plaquettes au subendothélium vasculaire, le vWF servant alors de lien entre le subendothélium et les plaquettes. Le rôle exact des motifs LR dans ces processus n'est pas clairement compris.

Les molécules à motif LR joueraient également un rôle dans l'activation qui suit la phase initiale. L'activation plaquettaire serait stimulée par une interaction entre le domaine intracytoplasmique du complexe GPIb/GPIX et le cytosquelette. La présence d'un site de phosphorylation dans la portion carboxy-terminale de $\operatorname{GPIb} \beta$ pourrait moduler cette interaction. La phosphorylation semble bloquer la polymérisation de l'actine et pourrait influencer le lien entre le complexe GPIb/GPIX et le compartiment intracytoplasmique des plaquettes [10].

Un récepteur de la famille des intégrines, connu sous le nom de complexe GPIIb-IIIa, prend ensuite le relai.

\section{La protéine OMgp (oligodendrocyte myelin glycoprotein)}

Cette glycoprotéine du système nerveux central est pourvue de sept motifs LR et d'un segment aminoterminal conservé avec d'autres protéines à motif LR [11]. Elle présente également quatre répétitions d'un module " serine/thréonine " impliqué dans des liaisons covalentes à des hydrates de carbone, qui est d'ailleurs retrouvé dans la molécule GPIb $\alpha$. OMgp est liée à la membrane plasmique par l'intermédiaire d'un glycosyl-phosphate-inositol (figure 2).

La myélinisation est un processus complexe qui requiert des interactions entre les membranes plasmiques des oligodendrocytes mais aussi avec les axones. De nombreuses protéines sont impliquées dans ce phénomène. OMgp, exprimée spécifiquement par les oligodendrocytes, pourrait, par sa structure et sa localisation membranaire, contribuer au mécanisme d'adhérence cellulaire nécessaire à la myélinisation [11]. En effet, outre les motifs LR connus pour interagir fortement avec d'autres modules protéiques, une sous-population des protéines OMgp porte un carbohydrate particulier, HNK-1, dont on sait qu'il intervient dans des mécanismes d'adhérence entre les cellules du système nerveux central [12]. Cependant, la fonction adhésive de OMgp n'a pas été démontrée.

\section{Les récepteurs TRK et autres kinases}

Certains récepteurs à tyrosine kinase comportent des motifs LR. Schneider et Schweiger [13] ont montré l'existence de trois motifs similaires aux 
LR (bien que non canoniques) dans la portion extracellulaire de TRK et TRKB, deux récepteurs neurogéniques à tyrosine kinase. En outre, les similitudes de séquence s'étendent aux cassettes riches en cystéines encadrant les modules LR en amino- et carboxy-terminal. La présence de ces modules LR suggère que TRK pourrait intervenir dans des phénomènes d'adhérence et non seulement transduire le signal d'une neurotrophine. Pulido a montré que D-TRK, l'homologue de TRK chez la drosophile, a des propriétés de molécule d'adhérence homophilique [14]. Dans ce cas précis, le processus d'adhérence provoque la stimulation de l'activité tyrosine kinase. D-TRK a donc la faculté d'être son propre ligand. Les motifs LR retrouvés dans les séquences de TRK et TRKB ne sont pas aussi clairement définis que pour D-TRK. Cela empêche donc d'établir une corrélation nette pour les molécules TRK, entre la présence de répétitions $L R$ et leur implication directe dans des processus d'adhérence.

Un autre récepteur à tyrosine kinase, $\mathrm{RYK}$, a été caractérisé récemment, et sa fonction est encore inconnue [15]. Il comporte deux motifs LR dans sa région extracellulaire.

Dans les exemples précédents le rôle exact des motifs LR n'est pas compris ou la fonction d'adhérence des molécules contenant les motifs LR n'est pas certaine. L'intervention de motifs LR dans le processus d'adhérence semble être plus directe dans les exemples suivants, empruntés à la drosophile.

\section{Les molécules d'adhérence à motifs $L R$ chez la drosophile}

La drosophile possède plusieurs classes de molécules d'adhérence [16]. Parmi elles, on trouve les molécules à motifs LR qui jouent des rôles divers.

La chaoptine et la morphogenèse des cellules photoréceptrices

Parmi les protéines à motifs LR, la chaoptine présente un certain nombre de particularités. Plus des quatre cinquièmes de la protéine sont constitués $\mathrm{m} / \mathrm{s} n^{\circ} 10$ vol. 9, octobre 93 par 41 motifs LR, regroupés en 3 blocs comprenant respectivement 10 , 14 et 17 répétitions [17-19]. L'extrémité carboxy-terminale de la protéine se lie au glycosyl-phosphatidylinositol de la bicouche lipidique et la chaoptine peut être libérée de son lieu de fixation par la phosphatidylinositol phospholipase C.

La chaoptine est exprimée spécifiquement au niveau de l'œil. L'œil de drosophile est une structure très organisée contenant 800 facettes hexagonales appelées ommatidies. Chaque ommatidie est constituée d'une colonne de huit cellules photoréceptrices (cellules R1 à R8) entourées d'un feuillet de pigment. Les cellules $\mathrm{R}$ comportent un axone qui s'étend de la base du corps cellulaire vers le ganglion optique. Le long de la colonne formée par les cellules $\mathrm{R}$ et bourgeonnant de celles-ci, se trouvent des organites photosensibles, constitués d'un empilement compact de microvillosités appelées rhabdomères $[20,21]$.

La différenciation des cellules $\mathrm{R}$ débute au $3^{e}$ stade larvaire. Au cours des quarante-huit premières heures du développement pupal, les cellules $\mathrm{R}$ projettent leur axone vers le ganglion optique en formation et des connexions synaptiques s'instaurent. Les rhabdomères se constituent alors et leur formation s'achèvera peu avant l'éclosion. L'expression de la chaoptine coïncide avec les étapes de différenciation des cellules $\mathrm{R}$. La présence de chaoptine est détectée au niveau des membranes des cellules $R$, au dernier stade larvaire et au stade adulte [22].

Après induction de mutations par irradiation aux rayons $\mathrm{X}$, un criblage immunologique a permis d'isoler des mutants du gène chaoptine [18]. L'analyse phénotypique révèle que ces mutations ne modifient pas l'aspect de l'œil et n'abolissent pas toute photosensibilité. La chaoptine n'intervient pas directement dans la phototransduction. En revanche, les mutants présentent des défauts conséquents dans l'ultrastructure de leurs rhabdomères. Le corps cellulaire des cellules $\mathrm{R}$ reste intact mais l'organite de photosensibilité est absent ou rudimentaire. Par ailleurs, les contacts membranaires entre les cellules $\mathrm{R}$ sont profondément affectés.
Le défaut dans la formation des rhabdomères, constaté pour les mutants du gène chaoptine, suggère que la chaoptine intervient dans leur organisation. Le développement normal d'un rhabdomère nécessite en particulier le compactage et l'alignement précis des microvillosités. La chaoptine, par un mécanisme d'adhérence, permettrait l'accolement de microvillosités adjacentes. Le processus d'adhérence a été testé dans des cellules de Schneider (cellules S2 d'embryon de drosophile) in vitro, et ces expériences montrent, en effet, le potentiel d'adhérence homophilique de la chaoptine [19] (figure 3).

Une expression précoce du gène chaoptine, précédant de trois ou quatre jours la formation du rhabdomère, indique qu'un autre rôle de la chaoptine, toujours basé sur des propriétés adhérentes, est néanmoins envisageable. L'accumulation de chaoptine le long des membranes plasmiques des cellules $\mathrm{R}$ avant l'extension des microvillosités pourrait assurer, par l'intermédiaire d'un mécanisme d'adhérence homophilique, le maintien d'un alignement correct des zones de contact entre ces cellules.

\section{La protéine Toll et la morphogenèse de l'embryon}

Ce polypeptide transmembranaire comporte dans la région extracytoplasmique dix-huit répétitions du domaine riche en leucines. Sa portion intracytoplasmique s'étend sur près de trois cents acides aminés et présente certaines similitudes de séquence avec le récepteur de l'IL1 [23]. Toll joue un rôle dans l'établissement de l'axe dorso-ventral de la drosophile [24-28]. La formation des structures dorso-ventrales d'un embryon de drosophile nécessite la transcription de onze gènes maternels [26, 28]. Les produits de ces gènes, en contrôlant la translocation nucléaire du produit du gène dorsal, créent un gradient de ce morphogène suivant l'axe dorso-ventral. Dans le noyau, dorsal active ou inhibe alors des gènes zygotiques spécifiques d'une région donnée.

Toll est l'un des gènes maternels qui participe à la mise en place de l'asy- 


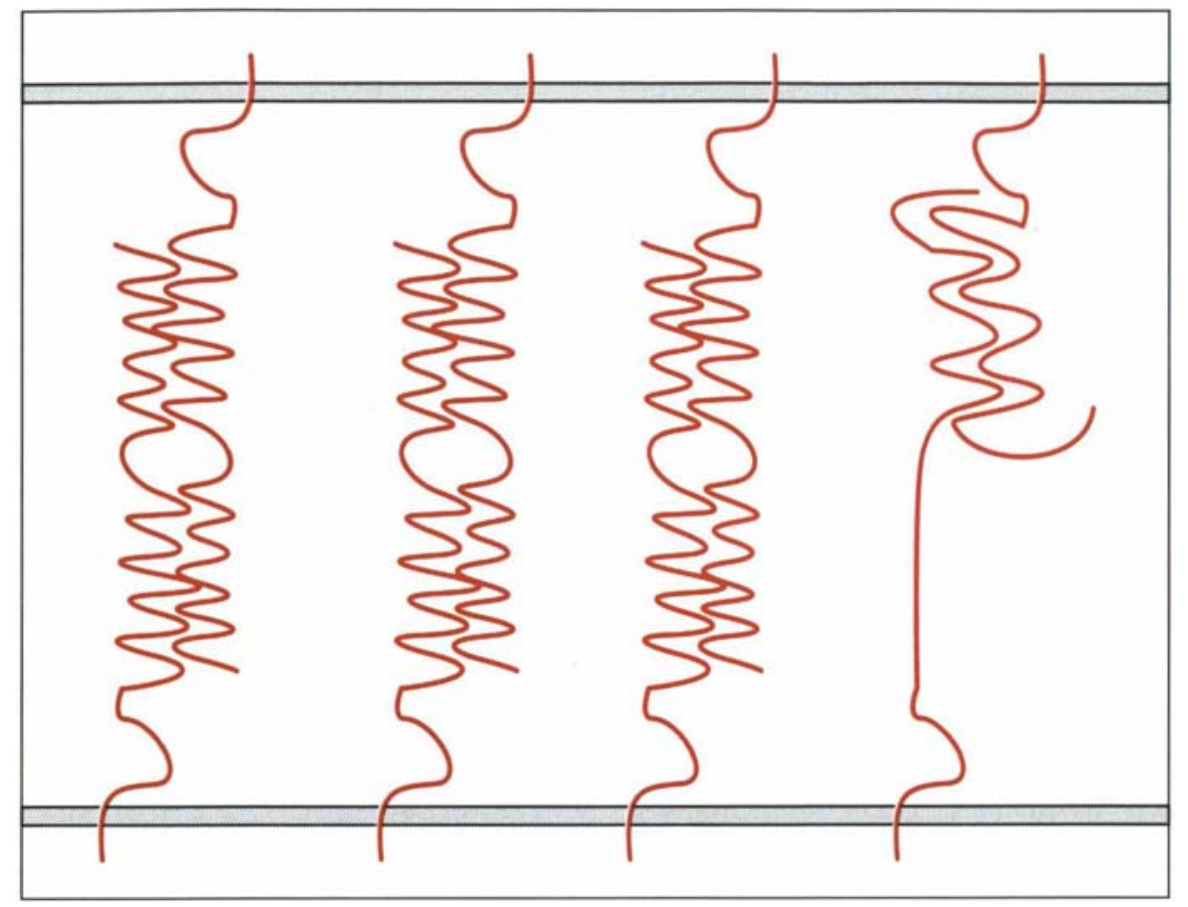

Figure 3. Représentation schématique de liaisons entre des molécules d'adhérence de deux cellules. Trois liaisons de type homophilique et une de type hétérophilique sont dessinées.

métrie dorso-ventrale. Il est exprimé au stade blastoderme syncytial alors que les noyaux ne sont pas encore cloisonnés en cellules. La protéine Toll est répartie de façon uniforme dans la membrane plasmique de l'embryon syncytial et, à ce stade, des protéines de l'espace périvitellin seraient nécessaires à la production du ligand, activant Toll ventralement. Des embryons issus d'une femelle porteuse d'une mutation récessive Toll seront essentiellement dorsalisés et dépourvus de toute structure latérale et ventrale. Toll est sous le contrôle de snake et easter et module elle-même l'expression d'autres gènes en aval, conduisant à la mise en place finale du gradient du facteur nucléaire dorsal. Les sérines protéases, codées par les gènes snake et easter, sont retrouvées dans l'espace périvitellin compris entre la membrane plasmique du syncytium et le chorion de l'œuf $[29,30]$. Le gène Toll codant pour une protéine membranaire occupe donc une position intermédiaire dans la voie de transduction qui aboutit à l'établissement du gradient dorso-ventral du facteur transcriptionnel dorsal. Plus tardivement dans le développement larvaire, l'étude du profil d'expression zygotique de Toll révèle une distribution spatiotemporelle complexe. Néanmoins, les transcrits de Toll se concentrent essentiellement dans des cellules subissant des invaginations $[24,25]$.

Toll intervient donc précocement dans la mise en place de l'asymétrie dorsoventrale en transduisant le signal d'un ligand localisé ventralement. Cette transduction de l'information jusqu'au noyau aboutit à la translocation nucléaire et à l'activation du morphogène dorsal dans les régions ventrales de l'embryon seulement.

Toll serait en même temps une molécule d'adhérence. Exprimée à la surface de cellules S2, Toll provoque l'adhérence cellulaire. Cette adhérence est de type hétérophilique (figure 3) [27]. In vivo l'expression de Toll n'est pas uniquement maternelle et Toll s'exprime également dans le zygote [25].

\section{La connectine et la jonction neuromusculaire}

Au sein de la famille des molécules d'adhérence à LR, la connectine pré- sente la particularité de s'exprimer dans deux types cellulaires distincts voués à interagir, la cellule musculaire et le motoneurone.

La connectine est une protéine de 648 résidus comprenant 10 motifs riches en leucines de 24 acides aminés chacun [31, 32]. A l'instar de la chaoptine (figure 2), elle ne contient pas de domaine transmembranaire, mais se fixe à la membrane plasmique par une liaison covalente de son extrémité carboxy-terminale à une ancre GPI.

Lors de la croissance du "cône " neuronal, de nombreuses molécules d'adhérence interviennent pour guider la progression d'un axone vers sa cellule cible [1]. Chez la sauterelle, des expériences d'ablation de muscle juste avant son innervation montrent que la croissance de l'axone du motoneurone requiert pour son cheminement la présence de la cellule cible [1]. De même, chez la drosophile, les précurseurs myoblastiques fournissent au motoneurone des informations déterminantes pour la mise en place d'une innervation spécifique. Les molécules impliquées dans la reconnaissance nerf/cellule cible au niveau des synapses sont pourtant encore largement inconnues. Dans le but de caractériser de tels acteurs, l'équipe de Goodman de l'université de Californie à Berkeley (CA, USA) a entrepris de cloner des gènes exprimés dans un sous-groupe de muscles avant leur innervation [30]. Cette démarche a permis l'isolement du gène de la connectine. La connectine pourrait contribuer à la reconnaissance ciblée d'un motoneurone spécifique pour une fibre musculaire particulière. En effet, elle est retrouvée, mais de façon transitoire, dans les cellules musculaires et dans les motoneurones destinés spécifiquement à les innerver. Sa disparition peu après la formation des synapses suggère que la connectine serait essentiellement utile dans la formation de la jonction neuromusculaire.

Il est envisageable que la connectine permette l'interaction spécifique entre l'axone et la fibre musculaire par un mécanisme d'adhérence cellulaire au niveau des synapses. En effet, l'expression d'ADNc de la connectine dans des cellules S2 induit une agrégation de ces cellules. Cette adhé- 
rence cellulaire provoquée par la connectine est de type homophilique (figure 3).

En dehors de muscles et de motoneurones spécifiques, l'expression de la connectine a été décelée au sein des systèmes nerveux central et périphérique dans certaines cellules gliales et une portion des axones. Ces observations indiquent que la connectine pourrait également jouer un rôle dans le guidage de certains cônes de croissance du système nerveux.

Nous avons récemment isolé, chez l'homme, un gène qui code pour une protéine transmembranaire à motifs LR qui ressemble beaucoup aux molécules d'adhérence de la drosophile, en particulier à la connectine et à Toll, mais sa fonction est encore inconnue [33]. La chaoptine et la connectine, ainsi que OMgp, sont ancrées à la membrane plasmique par une liaison covalente avec un glycosylphosphatidylinositol (GPI) localisé dans la couche lipidique [34, 35]. Ce type d'ancrage est souvent considéré comme facilitant les mouvements à la surface de la cellule ainsi que les interactions avec la membrane. Plasticité et mobilité sont ainsi peut-être des propriétés importantes de certaines molécules d'adhérence. De plus, la dégradation particulière de l'ancrage par GPI pourrait faciliter l'arrêt rapide de l'adhérence après un certain temps d'activité. Il a aussi été suggéré que les protéines ancrées à la membrane par un GPI pourraient transduire un signal par relargage de diacylglycérol après hydrolyse de la liaison par la phospholipase C. Certaines molécules à GPI sont par ailleurs complexées à une tyrosine kinase sous-membranaire.

\section{Connectine et Toll dans le dévelop- pement du muscle}

L'approche utilisée pour cloner le gène connectine a permis à Nose et al. d'isoler aussi le gène Toll, caractérisé par ailleurs (voir plus haut) [31]. Les études du profil d'expression de la connectine durant l'innervation musculaire de la drosophile ont été menées en parallèle pour Toll. Toll est exprimée à la surface de certaines fibres musculaires spécifiques, différentes de celles exprimant la connectine. En revanche, aucun motoneurone innervant ces muscles $\mathrm{m} / \mathrm{s} n^{\circ} 10 \mathrm{vol}$. 9, octobre 93 n'exprime Toll. On peut néanmoins envisager que les extrémités neurales présynaptiques possèdent à leur surface une molécule reconnaissant spécifiquement Toll puisque cette protéine fonctionne in vitro comme une molécule d'adhérence hétérophilique. Toll et la connectine, exprimées dans un nombre restreint de myoblastes sont donc de bons marqueurs pour l'étude de la formation de souspopulations de fibres musculaires. La fascicline III, appartenant à la famille des molécules d'adhérence à domaines immunoglobulin-like, s'exprime également transitoirement dans un sousgroupe de muscles en développement. Ces trois exemples montrent que des fibres musculaires individuelles sont marquées distinctement par des molécules d'adhérence spécifiques. Le schéma d'expression différentielle de Toll et de la connectine indique d'autre part que les myoblastes sont déjà engagés dans des destinées distinctes à un stade très précoce du développement musculaire [36]. Lors de ce processus, de rares cellules musculaires vont exprimer la connectine peu avant leur fusion avec les myoblastes progéniteurs exprimant déjà cette molécule d'adhérence. Il est envisageable que les précurseurs musculaires induisent par contact cellulaire certains myoblastes de leur environnement immédiat à transcrire des gènes spécifiques du muscle tels que le gène connectine ou Toll. Les cellules musculaires fondatrices entraîneraient ainsi les myoblastes environnants à suivre leur propre programme de développement. Toll et la connectine, par leurs propriétés d'adhérence, pourraient donc contribuer à propager un signal d'engagement dans un lignage de différenciation musculaire précis.

Les similitudes fonctionnelles entre Toll et la connectine ne sont pas surprenantes. Les mécanismes impliqués dans l'établissement de l'asymétrie dorso-ventrale de la drosophile sont analogues en certains points à ceux mis en jeu dans la différenciation des synapses [37]. Un point commun évident est l'importance des informations positionnelles apportées par des molécules de signalisation, qui conduit à l'expression sélective de certaines protéines à des endroits différents d'un syncytium.

\section{Conclusions}

Le motif riche en leucines est présent dans une très grande variété de molécules. Il joue certainement un rôle dans les interactions protéineprotéine. Le regroupement de quelques-unes de ces protéines dans cette revue repose sur un choix relativement arbitraire, si ce n'est pour le fait qu'elles sont toutes des molécules de surface et qu'elles semblent être plus spécifiquement impliquées dans des processus faisant intervenir des contacts entre cellules. En outre, les motifs riches en leucines représentent souvent une partie importante de ces protéines, bien que leur intervention exacte reste à préciser. Ils pourraient jouer le rôle simple de "bande Velcro " utile au maintien d'un contact, ou assurer un rôle bien précis au sein de molécules plus complexes capables de transmettre un signal

\section{Summary}

The fifth adhesion molecules family : the leucine rich motifs proteins

Numerous proteins contain a domain made of a variable number of leucine-rich repeats. These molecules exert various functions. Some of them, such as GPIb in humans, and chaoptin, Toll and connectin in Drosophila, appear to be adhesion molecules involved in cell-cell or cell-matrix interactions. GPIb is part of a complex which plays a central role in platelets function. Chaoptin, Toll and connectin are involved in developmental processes in Drosophila.

\section{Remerciements}

Les auteurs remercient les membres de leur équipe, en particulier F. Coulier et O. de Lapeyrière, pour les nombreuses discussions et critiques.

\section{TIRÉS A PART}

D. Birnbaum. 